\title{
Efecto del envase y del riego en vivero en el establecimiento de Pinus cooperi Blanco en dos condiciones de sitio
}

\author{
José Ángel Prieto-Ruiz¹, Pedro Antonio Domínguez-Calleros ${ }^{2}$, \\ Eladio H. Cornejo-Oviedo ${ }^{3}$ y José de Jesús Návar-Cháidez ${ }^{4}$
}

\section{RESUMEN}

\begin{abstract}
Se evaluó en campo, en dos sitios con características climáticas contrastantes: El Carmen, Durango y Santa Lucía, Pueblo Nuevo, Durango, el efecto de la calidad de planta de Pinus cooperi Blanco producida en vivero en envases de $80 \mathrm{~cm}^{3}$ y $170 \mathrm{~cm}^{3}$ de volumen y regada en la fase de preacondicionamiento cada 48, 96 y 168 horas. Los tratamientos se distribuyeron en un diseño experimental de bloques al azar, con un arreglo en parcelas subdivididas. Durante 18 meses se evaluó supervivencia, crecimiento en diámetro y altura, así como producción de fitomasa. La planta producida en el envase de $170 \mathrm{~cm}^{3}$ resultó superior estadísticamente $(p<0,05)$ en diámetro, con $9,26 \mathrm{~mm}$ y en producción total de fitomasa con $19,0 \mathrm{~g}$. Por otro lado, la planta regada cada 96 horas resultó superior estadísticamente $(p<0,05)$ en diámetro, con $9,17 \mathrm{~mm}$ y en producción de fitomasa con $17,9 \mathrm{~g}$. El tamaño de envase y la frecuencia de riegos no influyeron estadísticamente $(p>0,05)$ en la supervivencia y crecimiento en altura de las plantas. El factor sitio de plantación sólo tuvo diferencias significativas $(p<0,05)$ en la supervivencia y en el crecimiento en altura de las plantas. La supervivencia fue superior estadísticamente $(p<0,05)$ en el sitio El Carmen, Durango, con $85,6 \%$, mientras que el crecimiento en altura sobresalió en Santa Lucía, Pueblo Nuevo, Durango con $18,5 \mathrm{~cm}$.
\end{abstract}

PALABRAS CLAVE:

Calidad de planta, crecimiento, evaluación, manejo, plantación, supervivencia.

\section{ABSTRACT}

The objective was to evaluate seedling performance of Pinus cooperi Blanco planted at two contrasting field conditions: El Carmen, Durango, and Santa Lucía, Pueblo Nuevo, Dgo. The seedlings were produced under the following routines at the nursery: two sizes of container $\left(80 \mathrm{~cm}^{3}\right.$ and 170 $\mathrm{cm}^{3}$ ), and three irrigation frequencies (every 48,96 and 168 hours). A split-split plot experimental random block design was established at the two sites. Seedlings survival, height, diameter growth, and phytomass production were evaluated during 18 months. The container of $170 \mathrm{~cm}^{3}$ had a significantly superior effect $(p<0,05)$ on diameter growth and phytomass production, with averages of $9,26 \mathrm{~mm}$ and $19,0 \mathrm{~g}$, respectively. The 96 hours irrigation frequency had a significantly superior effect $(p<0,05)$ on diameter growth and phytomass production, with $9,17 \mathrm{~mm}$ and $17,9 \mathrm{~g}$, respectively. It was not found a statistical significance $(p>0,05)$ of container sizes and irrigation frequencies on seedling survival and height growth. The plantation site factor had a significance statistical $(p<0,05)$ on seedling survival and height growth. The highest survival rate, $85,6 \%$, was found at El Carmen, Durango, Dgo, and the largest height growth, $18,5 \mathrm{~cm}$, was recorded at Santa Lucía, Pueblo Nuevo, Dgo.

3 Departamento Forestal. Universidad Autónoma Agraria "Antonio Narro" Buenavista, Saltillo, Coahuila. ce: cornejo@forestal.org.mx

4 Centro de Investigación Interdisciplinaria para el Desarrollo Integral Rural (CIIDIR). IPN. Calle Sigma S/N. Fracc. 20 de Noviembre II. C.P. 34220. Durango, Dgo. 
KEY WORDS:

Seedling quality, growth, evaluation, management, plantation, survival.

\section{INTRODUCCIÓN}

Diversos factores influyen en la supervivencia y crecimiento de las plantas recién plantadas en campo, y entre ellos destacan la calidad del sitio, la fecha de plantación, las técnicas de preparación del sitio y de plantación, así como las características morfológicas (diámetro del cuello, altura, tamaño del sistema radical y relación parte aérearaíz, entre otras) y fisiológicas de las plantas (estado nutricional, estrés hídrico, niveles de carbohidratos, resistencia al frío y grado de lignificación, principalmente) (Beyeler, 1996; Birchler et al., 1998). Si la calidad de la planta es deficiente, la supervivencia y/o el crecimiento disminuye, lo que implica más tiempo para lograr que las reforestaciones prosperen y en consecuencia los costos de plantación aumentan (Landis, 2002).

Desafortunadamente, los logros alcanzados en los programas de reforestación realizados en México son poco alentadores debido a que se utiliza germoplasma de baja calidad genética, y a que existen deficiencias en los procesos de producción de planta, preparación del sitio, plantado y seguimiento a las plantaciones (CONAFOR, 2004a). Prueba de ello son los resultados de las evaluaciones realizadas entre 2001 y 2003, donde se encontraron niveles de supervivencia inferiores al $75 \%$ al año de plantado (CONAFOR, 2004b).

La calidad de la planta influye de manera determinante en el éxito de los programas de reforestación. A través del tiempo las técnicas de producción de planta han variado, buscándose siempre que los atributos morfológicos y fisiológicos de la planta estén acordes a las características edáficas y climáticas del sitio de plantación (Scagel et al., 1998). Sin embargo, en muchos casos las prácticas culturales se generalizan y en ocasiones existen deficiencias técnicas, lo que propicia que se produzca planta de baja calidad. Para producir planta con los atributos morfológicos y fisiológicos apropiados a las condiciones del sitio de plantación y a los objetivos del programa de reforestación, se requiere un adecuado proceso técnico respecto a la siembra, medios de crecimiento, nutrición, riego, prevención y control de plagas y enfermedades, y manejo del ambiente de producción, entre otros.

El tamaño de envase define el espacio de crecimiento de las plantas. Para optimizar costos se busca que tengan el menor volumen posible, sin afectar el crecimiento de las plantas (Bainbridge, 1994). El volumen del envase influye en el crecimiento del sistema radical, medio a través del cual las plantas absorben el agua y los nutrimentos (Mullan y White, 2002). Por otro lado, la restricción de humedad al medio de crecimiento de las plantas, previo a su establecimiento en campo, favorece su preacondicionamiento al reducir las tasas de crecimiento de las plantas, propiciar la aparición de la yema apical e incrementar la lignificación.

En el estado de Durango existen 21 viveros forestales, los cuales producen anualmente 4,5 millones de plantas del género Pinus. Sin embargo, la mayoría de los viveristas tienen poca claridad respecto al tamaño de envase que deben utilizar y carecen de experiencia sobre la aplicación de rutinas de riego que favorezcan la lignificación de las plantas para mejorar su calidad, lo que se ha reflejado en tasas de mortalidad mayores al $30 \%$ al año de plantado (Universidad Autónoma de Tlaxcala, 2002). Dado que el tamaño de envase y el preacondicionamiento de las plantas son aspectos importantes en la producción de 
planta y en su desarrollo posterior en el sitio de plantación, es necesario evaluar en campo la eficiencia de las prácticas de producción de planta en vivero.

\section{OBJETIVOS}

Evaluar la supervivencia y el crecimiento de planta de Pinus cooperi Blanco producida en dos tamaños de envase, regada bajo tres frecuencias en la fase de preacondicionamiento y plantada en dos sitios con características climáticas contrastantes.

\section{METODOLOGÍA}

\section{Condiciones de producción de la planta}

El estudio se efectuó en el vivero del Campo Experimental Valle del Guadiana, del Instituto Nacional de Investigaciones Forestales, Agrícolas y Pecuarias (INIFAP), en Durango, Durango, ubicado a $24^{\circ} 01^{\prime}$ LN y $104^{\circ} 44^{\prime} \mathrm{LO}$, y a $1830 \mathrm{~m}$ de altitud. La planta se produjo en un invernadero cubierto con plástico calibre 720 y malla sombra del $35 \%$.

Como sustrato se utilizó una mezcla compuesta por turba, vermiculita y agrolita al $55 \%, 24 \%$ y $21 \%$, respectivamente, a la que se le agregó el fertilizante granulado Multicote ${ }^{\mathrm{TM}}$ en dosis de $5 \mathrm{~kg} \mathrm{~m}^{-3}$, cuya formulación N-P-K (nitrógeno- $\mathrm{P}_{2} \mathrm{O}_{5}$ $\mathrm{K}_{2} \mathrm{O}$ ) fue de 15-07-15. Además, se fertilizó con Peters Professional ${ }^{\mathrm{TM}}$, en diferentes dosis, de acuerdo a cada fase de crecimiento que caracteriza a las plantas en vivero: a) fase de establecimiento: 75 187-151 ppm de N-P-K, b) fase de crecimiento rápido: $175-27-138$ ppm de N-P-K, y c) fase de preacondicionamiento: $75-$ 205-545 ppm de N-P-K. El fertilizante se aplicó cada 72 horas. El envase utilizado fue charola de poliestireno de 112 y 77 cavidades, con $80 \mathrm{~cm}^{3}$ y $170 \mathrm{~cm}^{3}$, respectivamente. Durante la fase de preacondicionamiento, la cual duró 45 días, el lote de plantas se dividió en tres grupos y se regó cada 48, 96 y 168 horas. Además, las plantas estuvieron en condiciones ambientales de intemperie.

\section{Preparación del sitio y plantación}

La preparación del sitio consistió en: a) eliminar la maleza con azadones para facilitar la plantación y evitar competencia inicial, b) delimitar las parcelas experimentales con cuerda, estacas y cal para ubicar los tratamientos de acuerdo al diseño experimental $y, c)$ hacer las cepas con talacho y pala, de $15 \mathrm{~cm}$ de diámetro y 20 $\mathrm{cm}$ de profundidad. El proceso de plantado consistió en acomodar la planta en forma manual en el centro de la cepa, poner tierra alrededor de la planta y apisonar levemente para eliminar espacios de aire. La plantación se realizó durante la primera quincena de agosto de 2002.

\section{Tratamientos evaluados}

Se evaluó en campo, en dos sitios, planta producida en vivero en dos tamaños de envase y regada en la fase de preacondicionamiento bajo tres frecuencias de riego, tal como se describe a continuación:

\section{Sitios de plantación}

A los ocho meses de edad de las plantas, éstas se plantaron en dos localidades: a) Predio Particular El Carmen, Durango, Durango, ubicado a $47 \mathrm{~km}$ de Durango, Durango y, b) Santa Lucía, Ejido Pueblo Nuevo, Pueblo Nuevo, Durango, localizado a $35 \mathrm{~km}$ del poblado El Salto, Pueblo Nuevo, Durango. Las características principales de dichos sitios se describen en la Tabla 1. 
Tabla 1. Ubicación, condiciones climáticas y edáficas de los sitios de plantación

\begin{tabular}{|c|c|c|}
\hline Característica & El Carmen, Dgo, Dgo. & $\begin{array}{l}\text { Santa Lucía, } \\
\text { Pueblo Nuevo, Dgo. }\end{array}$ \\
\hline Localización & $\begin{array}{r}23^{\circ} 54^{\prime} 57^{\prime \prime} \mathrm{N} \\
104^{\circ} 56^{\prime} 58^{\prime \prime} \mathrm{W}\end{array}$ & $\begin{array}{l}23^{\circ} 36^{\prime} 10^{\prime \prime} \mathrm{N} \\
105^{\circ} 32^{\prime} 10^{\prime \prime} \mathrm{W}\end{array}$ \\
\hline Altitud (m) & 2383 & 1924 \\
\hline Pendiente (\%) & Menor a 3 & 15 \\
\hline Precipitación media anual (mm) & 914 & 1,270 \\
\hline Temperatura media anual $\left({ }^{\circ} \mathrm{C}\right)$ & 12,6 & 16,0 \\
\hline $\mathrm{pH}^{*}$ & 7,38 & 7,57 \\
\hline Materia orgánica $(\%)^{*}$ & 1,84 & 4,62 \\
\hline Nitrógeno $\left(\mathrm{N}-\mathrm{NO}^{3} \mathrm{mg} \mathrm{kg}^{-1}\right)^{*}$ & 10,2 & 9,3 \\
\hline Fósforo $\left(\mathrm{mg} \mathrm{kg}^{-1}\right)^{*}$ & 6 & 25 \\
\hline Potasio $\left(\mathrm{mg} \mathrm{kg}^{-1}\right)^{*}$ & 360 & 538 \\
\hline Arena $(\%)^{*}$ & 47 & 31 \\
\hline $\operatorname{Limo}(\%)^{*}$ & 39 & 30 \\
\hline Arcilla $(\%)^{*}$ & 14 & 39 \\
\hline Clase textural ${ }^{*}$ & Franco & Franco arcilloso \\
\hline
\end{tabular}

Tamaños de envase. Se utilizaron dos tamaños de envase, contenidos en charolas de poliestireno: a) envase de $80 \mathrm{~cm}^{3}$ de volumen, con $10,4 \mathrm{~cm}$ de largo y $3,6 \mathrm{~cm}$ de diámetro superior, y b) envase de $170 \mathrm{~cm}^{3}$ de volumen, con $15,6 \mathrm{~cm}$ de largo y $4,3 \mathrm{~cm}$ de diámetro superior. Se considera al envase de menor volumen como control, al ser el más utilizado en la producción de planta en los viveros de Durango.

Frecuencias de riego. Durante 45 días, en la fase de preacondicionamiento de las plantas, se regó en tres intervalos de tiempo: a) cada 48 horas, equivalente a un contenido de humedad del sustrato (CH) entre $335 \%$ y $173 \%$, a este tratamiento se le consideró como control al ser la forma tradicional de riego que aplican los viveristas en el área de influencia del estudio, b) cada 96 horas, con un $\mathrm{CH}$ de $333 \%$ a $87 \%$, y c) cada 168 horas, con un $\mathrm{CH}$ de $346 \%$ a $69 \%$, tiempo donde empezó a manifestarse marchitez inicial en la yema apical de las plantas. Los $\mathrm{CH}$ máximos correspondieron a la condición de saturación de humedad del sustrato al inicio de los ciclos de estrés hídrico, en tanto que los $\mathrm{CH}$ mínimos se obtuvieron al finalizar los ciclos de estrés. Después de terminar cada ciclo el sustrato se mantuvo regado hasta saturación durante 24 horas.

\section{Diseño experimental}

Se utilizó un diseño experimental de bloques completamente al azar con un arreglo de subparcelas divididas. Se estableció un diseño en bloques debido a la pendiente del $15 \%$ existente en uno de los sitios. La parcela grande correspondió a los sitios de plantación, la mediana a las frecuencias de riegos y la pequeña a los tamaños de envase. Cada unidad experimental estuvo compuesta por 25 plantas, con cuatro repeticiones por tratamiento. 


\section{Modelo estadístico}

El modelo estadístico utilizado fue:

$Y_{i j k l}=\mu+\beta_{i}+\tau_{j}+(\beta \tau)_{i j}+\eta_{k}+(\tau \eta)_{j k}+$

$-(\beta \eta)_{i k}+\delta_{l}+(\tau \delta)_{j l}(\eta \delta)_{k l}+(\tau \eta \delta)_{j k l}+\varepsilon_{i j k l}$

Dónde: $Y_{i j k l}=$ variables respuesta, $\mu=$ promedio general de las diferentes fuentes de variación, $\beta_{i}=$ efecto del i-ésimo bloque, $\tau_{j}=$ efecto del j-ésimo sitio de plantación, $\eta_{k}=$ efecto de la k-ésima frecuencia de riego, $\delta_{l}=$ efecto del l-ésimo tamaño de envase, $\varepsilon_{i j k l}=$ error aleatorio. Las combinaciones de letras representan las interacciones con los bloques para los diferentes errores y entre los factores evaluados.

Los datos se analizaron mediante análisis de varianza a través del paquete Statistical Analysis System (SAS), utilizándose el procedimiento PROC GLM. Cuando se encontraron diferencias significativas entre tratamientos $(p<0,05)$, se realizaron pruebas de comparación de medias de Tukey.

\section{Variables evaluadas}

A partir de la plantación, agosto de 2002, se realizaron tres evaluaciones cada seis meses (febrero de 2003, agosto de
2003 y febrero de 2004). Las variables registradas fueron: a) supervivencia; para normalizar los datos, éstos se transformaron a la función arco seno, b) altura de la parte aérea, se midió de la base del tallo hasta la yema apical con una regla graduada en milímetros, c) diámetro del cuello, se evaluó en la base del tallo con un vernier digital con aproximación a centésimas de milímetro y, d) producción de fitomasa, estimada sólo en la evaluación final, a los 18 meses del plantado. Para ello, en forma aleatoria se extrajeron cuatro plantas por unidad experimental, las cuales se secaron durante 72 horas en una estufa a $72^{\circ} \mathrm{C}$. La determinación del peso seco se realizó en una balanza digital con aproximación a centésimas de gramo.

\section{RESULTADOS Y DISCUSIÓN}

Los resultados de las evaluaciones de campo incluyen el efecto acumulado de la fase de vivero, de los factores tamaños de envase (dos niveles) y frecuencia de riegos (tres niveles); por ello, en las variables evaluadas, con excepción de la supervivencia, existen diferencias estadísticas $(p<0,05)$ al finalizar la fase de vivero, tal como se muestra en los resultados y que corresponde a la condición de la planta al plantarse en campo (Tabla 2).

Tabla 2. Valores medios de las variables evaluadas al finalizar la etapa de vivero, a los nueve meses de edad de Pinus cooperi Blanco. Agosto de 2002.

\begin{tabular}{lccccc}
\hline \multicolumn{1}{c}{ Factor } & $\begin{array}{c}\text { Altura } \\
(\mathrm{cm})\end{array}$ & $\begin{array}{c}\text { Diámetro del cuello } \\
(\mathrm{mm})\end{array}$ & $\begin{array}{c}\text { Peso seco } \\
\text { parte aérea }(\mathrm{g})\end{array}$ & $\begin{array}{c}\text { Peso seco } \\
\text { raiz }(\mathrm{g})\end{array}$ & $\begin{array}{c}\text { Peso seco total } \\
(\mathrm{g})\end{array}$ \\
\hline $\begin{array}{l}\text { Envase (Volumen): } \\
170 \mathrm{~cm}^{3}\end{array}$ & $8,85 \mathrm{a}$ & $4,47 \mathrm{a}$ & $1,55 \mathrm{a}$ & $0,74 \mathrm{a}$ & $2,29 \mathrm{a}$ \\
$\quad 80 \mathrm{~cm}^{3}$ & $8,12 \mathrm{~b}$ & $4,04 \mathrm{~b}$ & $1,28 \mathrm{~b}$ & $0,49 \mathrm{~b}$ & $1,77 \mathrm{~b}$ \\
$\begin{array}{l}\text { Riego (Frecuencia): } \\
\text { Cada 48 horas }\end{array}$ & $9,05 \mathrm{a}$ & $4,52 \mathrm{a}$ & $1,58 \mathrm{a}$ & $0,74 \mathrm{a}$ & $2,32 \mathrm{a}$ \\
Cada 96 horas & $8,35 \mathrm{ab}$ & $4,19 \mathrm{~b}$ & $1,35 \mathrm{~b}$ & $0,58 \mathrm{~b}$ & $1,94 \mathrm{~b}$ \\
Cada 168 horas & $8,05 \mathrm{~b}$ & $4,05 \mathrm{~b}$ & $1,32 \mathrm{~b}$ & $0,52 \mathrm{~b}$ & $1,84 \mathrm{~b}$ \\
\hline
\end{tabular}




\section{Tamaños de envase}

La supervivencia no mostró diferencias significativas $(p>0,05)$ en las tres evaluaciones realizadas (Figura 1), debido al efecto de los tamaños de envase utilizados en vivero. Aunque la supervivencia durante el período de evaluación disminuyó cerca de $24 \%$ en ambos tamaños de envase, las causas de mortalidad no son atribuibles al tamaño del cepellón con que se plantaron, ello a pesar de que los envases utilizados tuvieron $90 \mathrm{~cm}^{3}$ de diferencia en volumen. Un factor que pudo resultar adverso para la planta producida en ambos envases, es haber plantado tardíamente (principios de agosto) debido a que el período de lluvias se retrasó.

Los resultados de supervivencia coinciden con los obtenidos por Cañellas et al. (1999), quienes encontraron que el tamaño de envase no influyó en la supervivencia de Pinus pinea $L$. después de dos años de plantado. En cambio, South et al. (2005), indican que el tamaño de envase $\left(90 \mathrm{~cm}^{3}\right.$ a $120 \mathrm{~cm}^{3}$ y a raíz desnuda), con que se produjo Pinus palustris $P$. Mill. en vivero, influyó en la supervivencia de las plantas en campo con los mejores resultados en los envases de mayor volumen. Por su parte, Moreno (2000) señala que, debido a los altos índices de supervivencia que se han obtenido en los diferentes tipos de envase disponibles en el mercado, el énfasis de su funcionalidad se ha enfocado más a los índices de crecimiento de las plantas.

Con relación al crecimiento en altura, en la fase de vivero existieron diferencias significativas $(p<0,05) ;$ sin embargo, en el sitio de plantación dicho efecto sólo duró hasta los 12 meses del plantado (Figura 2). El incremento en altura durante los últimos seis meses de evaluación disminuyó notablemente en la planta producida en el envase de 170 $\mathrm{cm}^{3}$, debido posiblemente a que la planta utilizó los carbohidratos para crecer en diámetro, variable que sobresalió en la planta producida en dicho envase. Se ha observado que en sitios con caracterís-

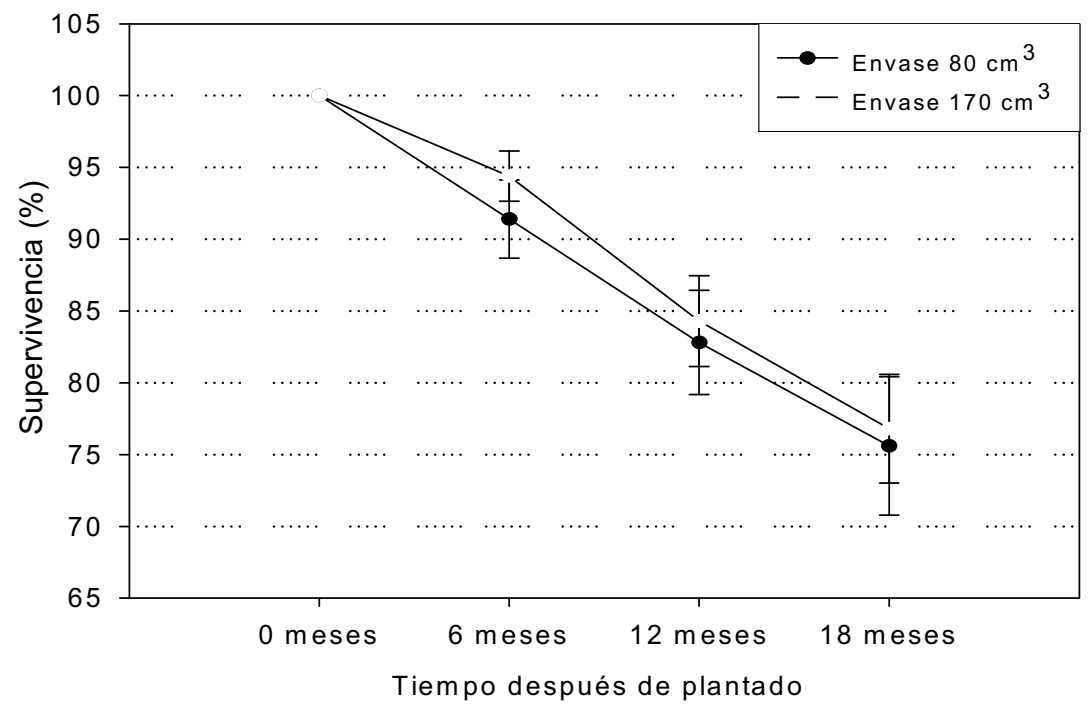

Figura 1. Supervivencia de planta producida en dos tamaños de envase a los 6,12 y 18 meses de plantada. 
ticas similares a las del experimento, Pinus cooperi Blanco crece en altura menos de $10 \mathrm{~cm}$ por año durante los primeros dos años de plantado y posteriormente las tasas se incrementan más rápido; sin embargo, la planta es robusta lo cual favorece su adaptación al sitio de plantación.

Por otro lado, el diámetro del cuello también presentó diferencias significativas $(p<0,05)$, efecto manifestado desde la fase de vivero y que continuó en campo durante el período de evaluación, con superioridad en la planta producida en el envase de $170 \mathrm{~cm}^{3}$ (Figura 3). Las diferencias en diámetro, entre tratamientos, se mantuvieron aproximadamente en $10 \%$ a los 6 meses y 12 meses de plantado y se incrementaron a $19 \%$ a los 18 meses. Estos resultados resaltan la importancia que tiene el tamaño del sistema radical en el crecimiento en diámetro de las plantas, variable que tiene relación con la robustez y supervi- vencia de las plantas en campo (Mexal y Landis, 1990).

La producción de fitomasa de la raíz, de la parte aérea y total, a los 18 meses del plantado, tuvo efectos significativos $(p<0,05)$ debido al factor tamaño de envase, con los mejores resultados en la planta producida en el envase de $170 \mathrm{~cm}^{3}$ (Figura 4), al superar en cerca del $30 \%$ a la del envase de $80 \mathrm{~cm}^{3}$ con diferencias de $1,67 \mathrm{~g}$ en la raíz, de $3,11 \mathrm{~g}$ en la parte aérea y de $5,50 \mathrm{~g}$ en la fitomasa total. Esta variable es importante debido a que cerca del $75 \%$ de la fitomasa está compuesta por carbohidratos, almacenados en las raíces y el tallo, los cuales son originados por la fotosíntesis de las plantas, esto hace que la producción de fitomasa sea un indicador de la eficiencia en el crecimiento de las plantas (Carlson y Miller, 1990).

Los resultados de las variables evaluadas ubican al envase de $170 \mathrm{~cm}^{3}$ como el más favorable para producir planta,

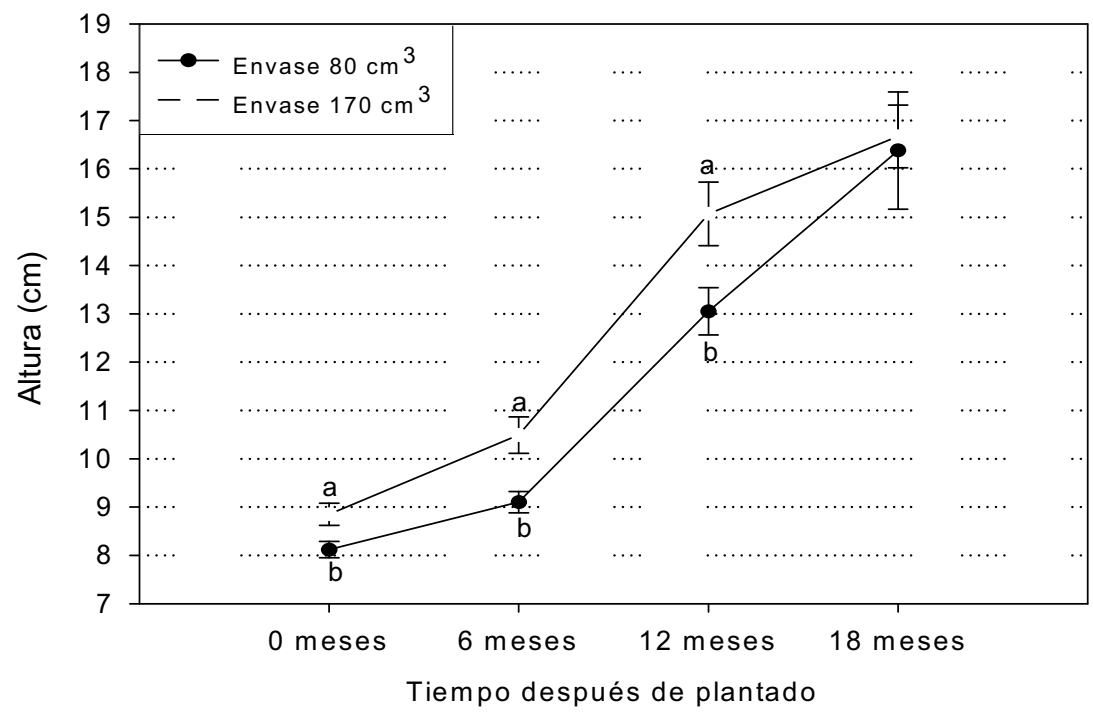

Figura 2. Crecimiento en altura de planta producida en dos tamaños de envase a los 6 , 12 y 18 meses de plantada. Letras diferentes para la misma fecha de evaluación indican diferencias significativas (Tukey, $p<0,05$ ). 


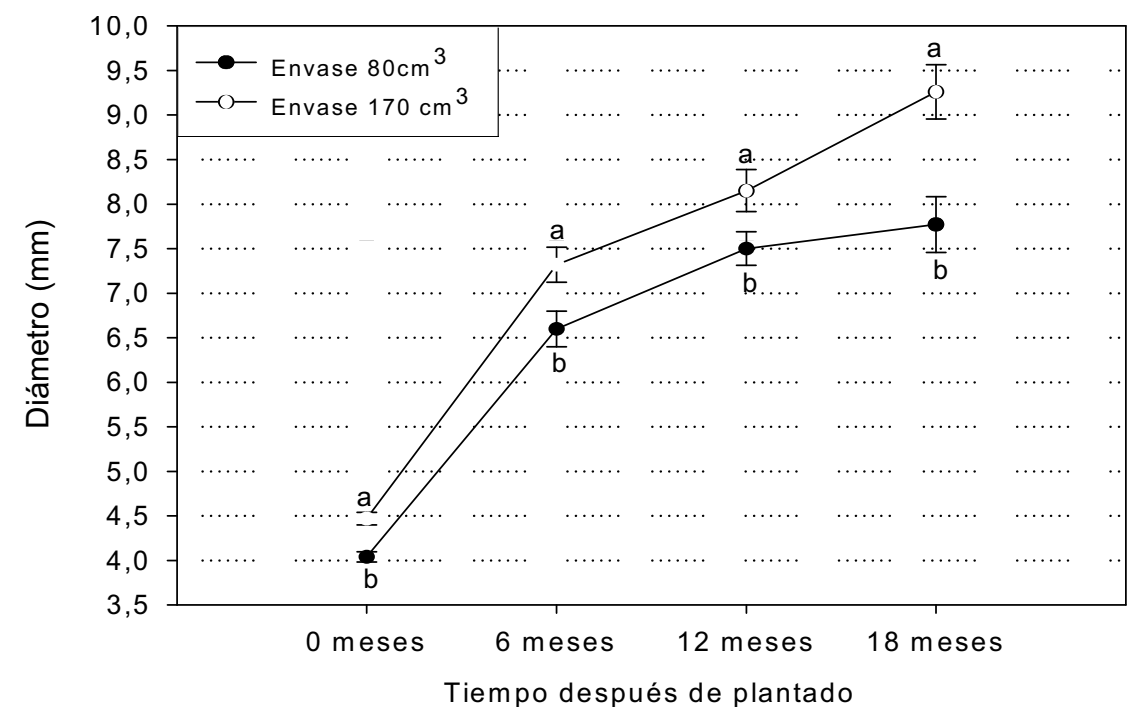

Figura 3. Crecimiento en diámetro del cuello de planta producida en dos tamaños de envase a los 6,12 y 18 de plantada. Letras diferentes para la misma fecha de evaluación significan diferencias estadísticas (Tukey, $p<0,05$ ).

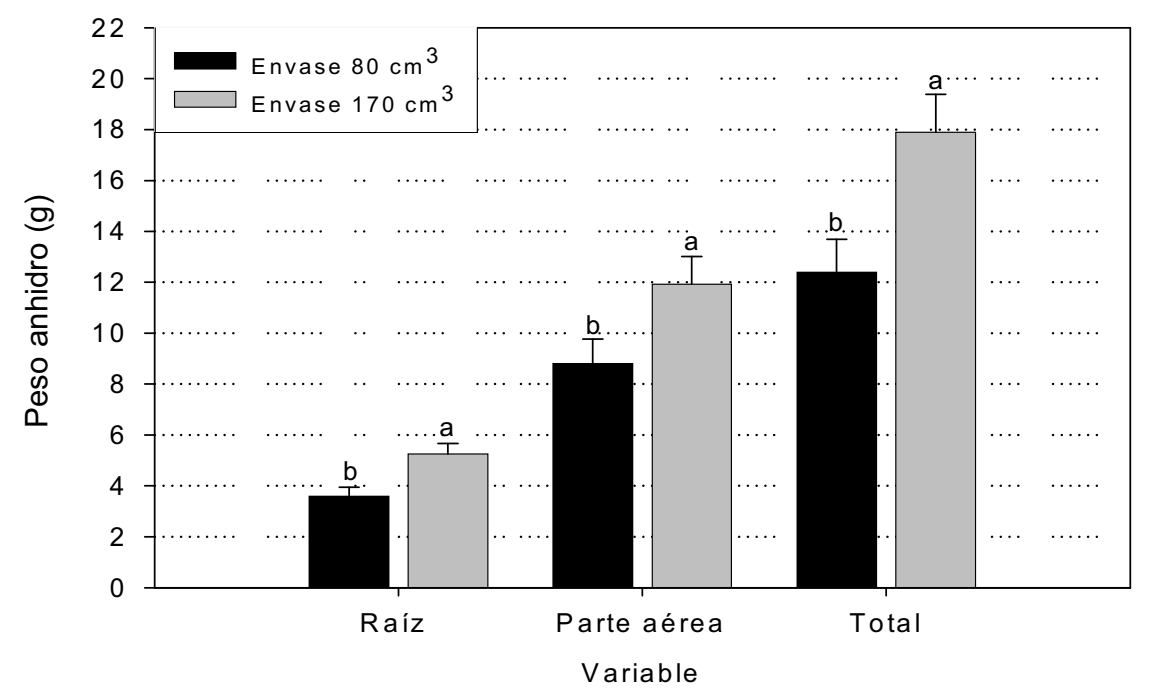

Figura 4. Producción de fitomasa en Pinus cooperi Blanco por tamaño de envase a los 18 meses del plantado (febrero de 2004). Letras diferentes para la misma variable indican diferencias significativas (Tukey, $p<0,05$ ) 
lo cual se reflejó en un mayor crecimiento en los sitios de plantación. Al respecto, Cañellas et al. (1999), Domínguez et al. (2000) y Villar et al. (2001), evaluaron en campo planta producida en envases de 50 $\mathrm{cm}^{3}$ a $700 \mathrm{~cm}^{3}$ y coinciden en que los envases de mayor volumen favorecen más el crecimiento de las plantas debido a que el sistema radical tiene mayor área de contacto con el suelo, lo que favorece la absorción de humedad y de nutrimentos. En ese sentido, Barnett y McGilvray (2000), así como Barnett (2002) indican que esta situación es importante cuando las condiciones de humedad y de calidad del suelo son adversas en el sitio de plantación.

Sin embargo, la selección del envase además de considerar su funcionalidad en el crecimiento biológico de las plantas, debe tomar en cuenta los costos de producción. Con el envase de $170 \mathrm{~cm}^{3}$ los costos de producción superan en cerca de $20 \%$ al envase de $80 \mathrm{~cm}^{3}$ (Aldana y Aguilera, 2002). A pesar de ello, el envase de
$170 \mathrm{~cm}^{3}$ es buena alternativa para producir planta ya que favoreció más su crecimiento en vivero, efecto reflejado en el sitio de plantación, objetivo final de cualquier programa de reforestación.

\section{Frecuencia de riegos}

La supervivencia de las plantas no mostró diferencias estadísticas $(p>0,05)$ como resultado de las frecuencias de riego aplicadas en vivero durante la fase de preacondicionamiento (Figura 5), lo cual pudo deberse a que el tiempo de aplicación de los tratamientos en vivero (45 días) resultó insuficiente para influir en la lignificación de las plantas, aspecto que favorece la supervivencia en los sitios de plantación, principalmente donde la disponibilidad de humedad es un factor crítico. Aunque la diferencia en supervivencia, a los 18 meses del plantado, resultó cercana al $10 \%$, entre el tratamiento con riego cada 168 horas y los

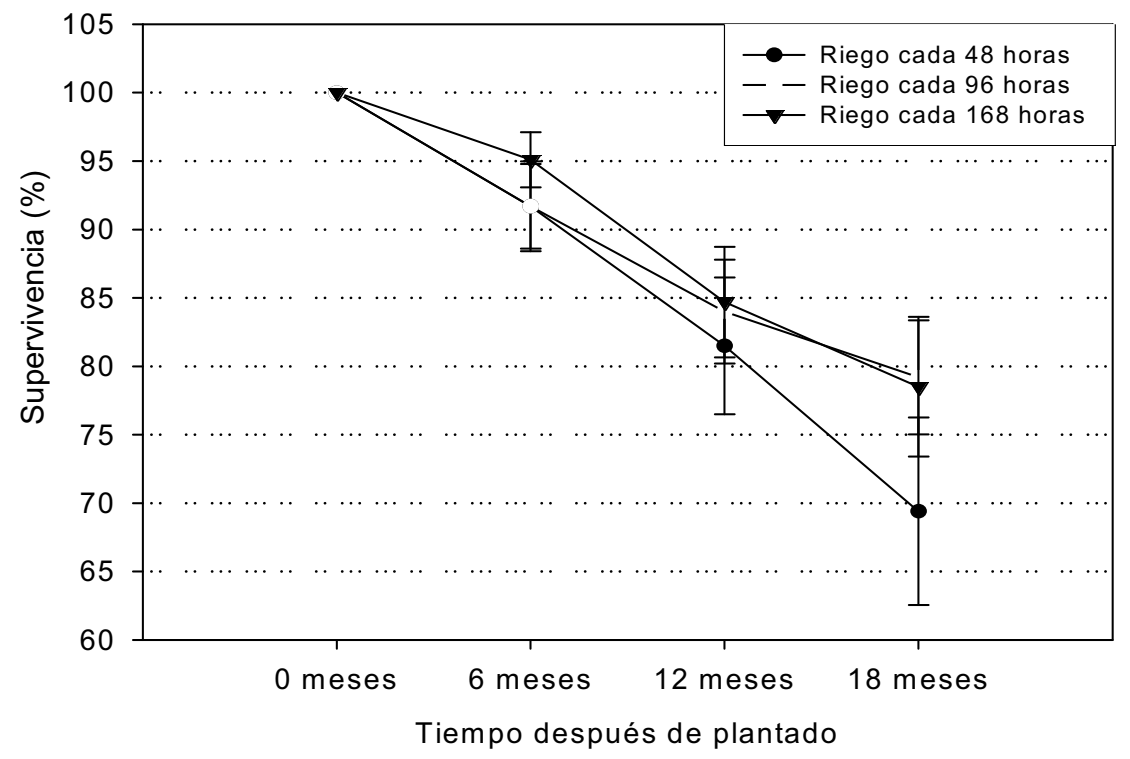

Figura 5. Supervivencia de Pinus cooperi Blanco por régimen de riego en vivero en la fase de preacondicionamiento a los 6,12 y 18 meses de plantado. 
tratamientos regados cada 48 y 96 horas en vivero, la inexistencia de diferencias estadísticas $(p>0,05)$ se debió a la gran variación que existió entre las parcelas de los mismos tratamientos.

Pese a que la planta producida en vivero manifestó diferencias en el crecimiento en altura (Figura 6), dicho efecto desapareció en campo a los seis meses de plantada y volvió a manifestarse a los 12 meses, con superioridad de la planta regada cada 96 horas. Sin embargo, en la evaluación final nuevamente los crecimientos en altura, con valores que fluctuaron entre $16,0 \mathrm{~cm}$ y $17,0 \mathrm{~cm}$, resultaron similares estadísticamente $(p<0,05)$. Al respecto, es posible que el lento crecimiento que tiene Pinus cooperi Blanco después de plantado sea la causa por la cual no existieron diferencias en forma determinante entre los tratamientos evaluados.
En cambio, la variable diámetro del cuello mostró diferencias estadísticas $(p<0,05)$ a los 6,12 y 18 meses del plantado, debido al estrés hídrico a que fueron sometidas las plantas en vivero. Sin embargo, el tratamiento que mejor favoreció el crecimiento en diámetro del cuello, riego cada 48 horas, fue superado en campo por la frecuencia de riego cada 96 horas, la cual sobresalió a partir de los seis meses del plantado (Figura 7). Las razones de estos resultados pueden deberse a que el riego cada 96 horas favoreció la preadaptación de las plantas a las condiciones del sitio donde se plantaron, ya que en campo la humedad disponible fue irregular; además, cuando se plantó, agosto de 2002, el material vegetativo estaba en pleno crecimiento activo, lo que hizo que las plantas regadas cada 48 horas, utilizadas como control, resintieran más el efecto del riego aplicado sin restricción en vivero.

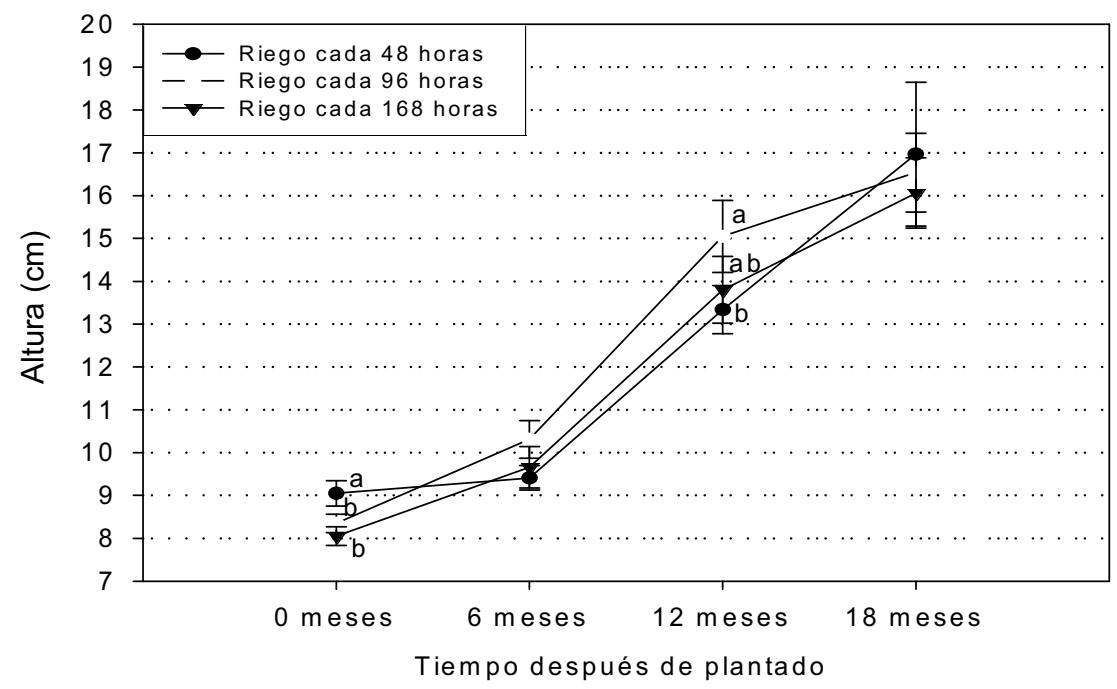

Figura 6. Crecimiento en altura de las plantas por régimen de riego en vivero en la fase de preacondicionamiento a los 6,12 y 18 meses del plantadas. Letras diferentes para la misma fecha de evaluación indican diferencias significativas (Tukey, $p<0,05$ ) 


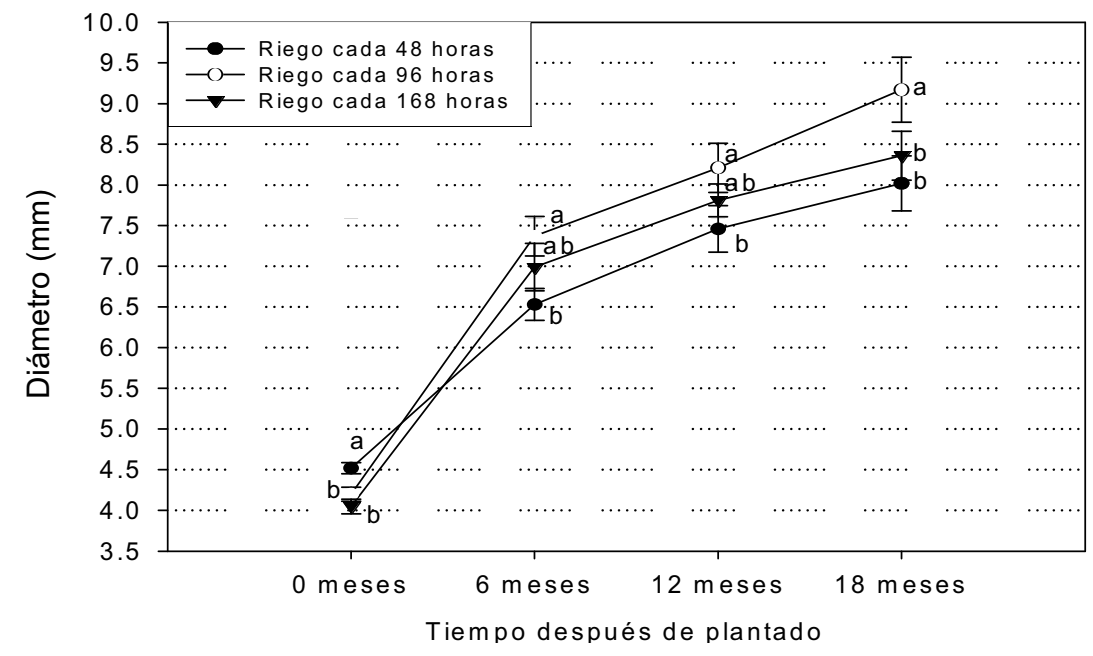

Figura 7. Crecimiento en diámetro del cuello por régimen de riego en vivero en la fase de preacondicionamiento a los 6,12 y 18 meses del plantado. Letras diferentes para la misma fecha de evaluación indican diferencias significativas (Tukey, $p<0,05$ ).

La producción de fitomasa del sistema radical, de la parte aérea y total de las plantas a los 18 meses del plantado, manifestó diferencias significativas $(p<0,05)$ como resultado de las frecuencias de riego utilizadas en la fase de preacondicionamiento en vivero, con los mejores resultados cuando se regó cada 96 horas (Figura 8). Los resultados de producción de fitomasa coinciden en forma consistente con los obtenidos en la variable diámetro; al respecto, se considera que la restricción de humedad en vivero propició una disminución en el crecimiento en diámetro y en producción de fitomasa. Sin embargo, una vez que se plantó en campo, en condiciones similares para los tres tratamientos, el crecimiento de las plantas se activó nuevamente y superó al de las plantas regadas cada 48 horas, el cual resultó superior en vivero; incluso las diferencias en producción de fitomasa se incrementaron conforme transcurrió el tiempo.
Los resultados anteriores resaltan la importancia que tiene el manejo del riego durante la fase de preacondicionamiento de las plantas, donde deben evitarse excesos de humedad para evitar que la planta crezca sin restricción y le afecte durante su establecimiento en campo, al plantarse cuando están en pleno crecimiento activo en verano, época del año en que ocurre la temporada de lluvias.

Para que el preacondicionamiento de las plantas en vivero sea adecuado, es necesario aplicar en forma integrada diversas prácticas culturales, tales como: reducción de humedad, modificación de la rutina de fertilización (Boivin et al., 2002), con disminución del nitrógeno y aumento del potasio, así como eliminación del efecto de invernadero para que la planta se adapte a las condiciones ambientales donde será plantada. Para este caso el manejo de la planta en vivero se realizó bajo las condiciones indicadas anteriormente. 


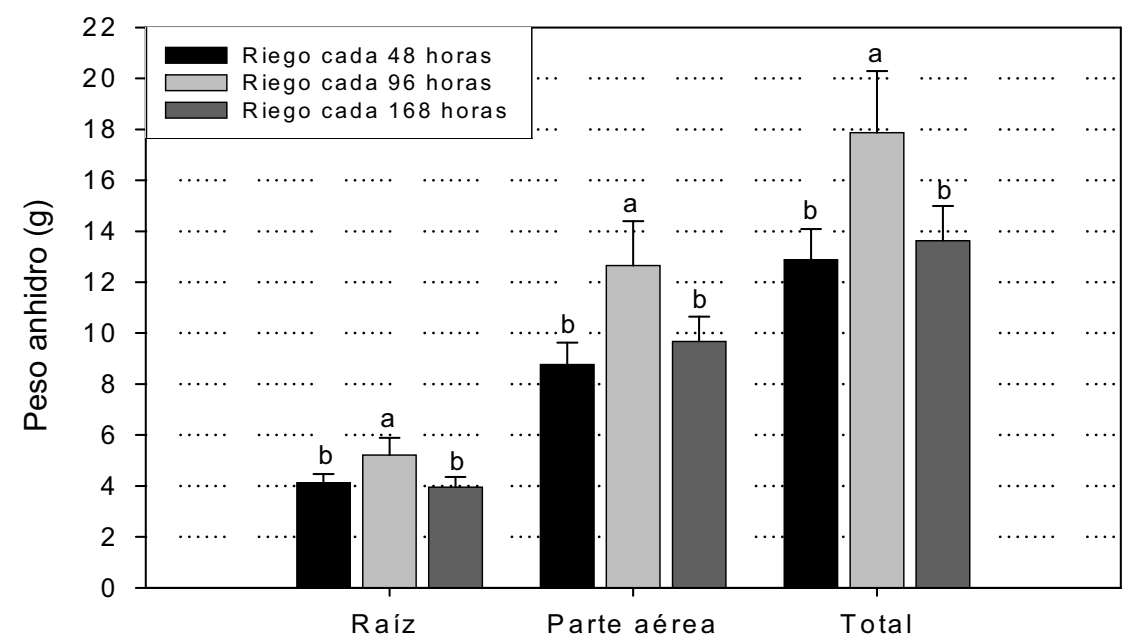

Figura 8. Producción de fitomasa en Pinus cooperi Blanco por régimen de riego en vivero a los 18 meses de plantado (febrero de 2004). Letras diferentes para la misma variable indican diferencias significativas (Tukey, $p<0,05$ ).

La aplicación del estrés hídrico a planta del género Pinus se ha realizado en diversas especies y en diferentes condiciones, con resultados diversos (Villar et al., 1997; Cetina et al., 2002; Martínez et al., 2002; Cornejo y Emmingham, 2003; Prieto et al., 2004). Por ello, debe evitarse generalizar esta práctica cultural, ya que las necesidades fisiológicas de las plantas varían en función de la especie, de las condiciones de producción y de las características del sitio de plantación. Esto crea la necesidad de generar sistemas de manejo en función de las condiciones de producción de cada vivero y de las características de los sitios de plantación.

\section{Condiciones del sitio}

La supervivencia de las plantas mostró diferencias significativas $(p<0,05)$ a los 6,12 y 18 meses del plantado debido al efecto de los sitios de plantación, con los porcentajes más altos en el sitio ubicado en El Carmen, Durango,
Dgo., con $20,5 \%$ de diferencia entre ambos sitios a los 18 meses del plantado (Figura 9).

Las diferencias en supervivencia pueden deberse básicamente a las condiciones climáticas que prevalecieron en ambos sitios. En Santa Lucía, Pueblo Nuevo, Dgo., a pesar de que la precipitación anual fue mayor, con $1270 \mathrm{~mm}$, el período de lluvias al igual que en El Carmen, Durango, Dgo., se concentra sólo de junio a septiembre; en el resto del año la humedad es escasa, salvo diciembre y enero con lluvias ocasionales. Asimismo, en el sitio de Santa Lucía, Pueblo Nuevo, Dgo., la exposición hacia la vertiente del Océano Pacífico hace que el sitio sea más cálido, con $16^{\circ} \mathrm{C}$ de temperatura media anual, y en consecuencia la humedad del suelo se pierde más rápidamente y existe mayor evapotranspiración del follaje de las plantas.

La variable altura también mostró diferencias significativas $(p<0,05)$ debido al efecto del sitio de plantación a partir de los seis meses del plantado (Figura 10), con 


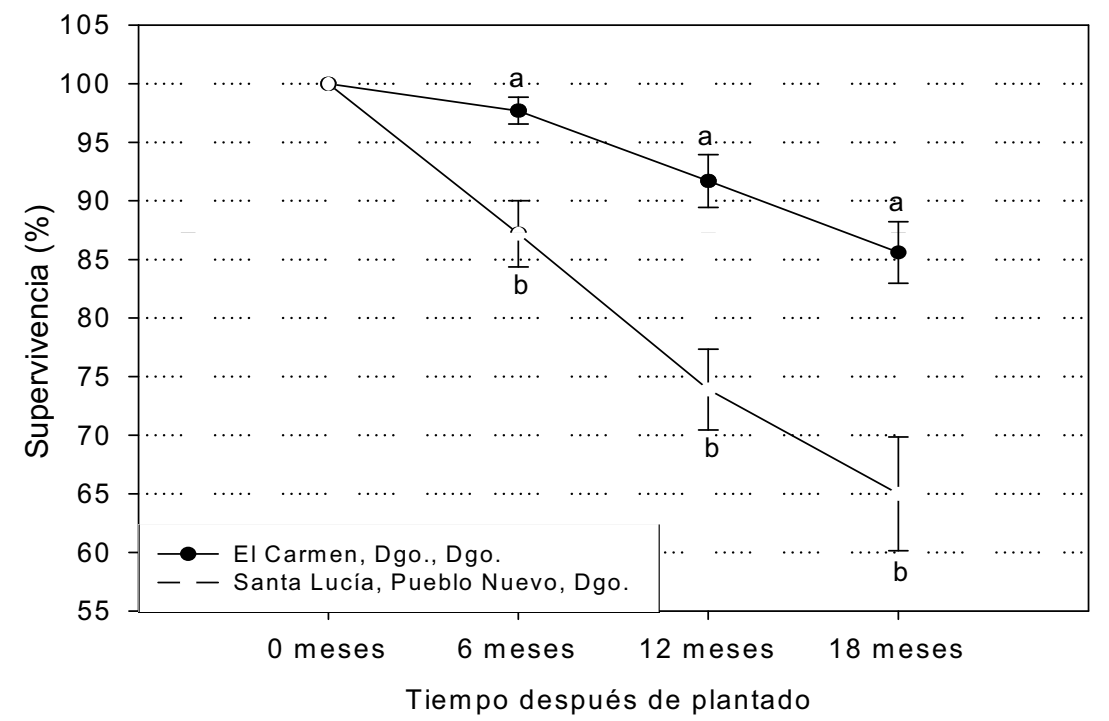

Figura 9. Supervivencia de Pinus cooperi Blanco en El Carmen, Durango, Dgo. y Santa Lucía, Pueblo Nuevo, Dgo. a los 6, 12 y 18 meses de plantado. Letras diferentes para la misma fecha de evaluación indican diferencias significativas (Tukey, $p<0,05$ ).

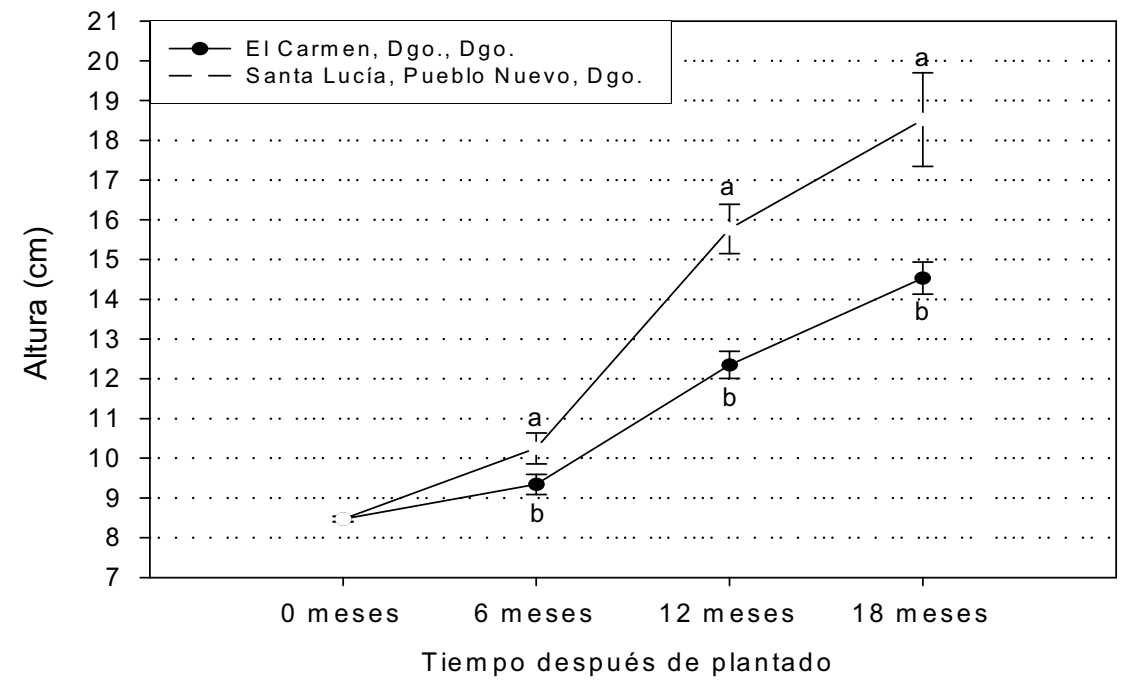

Figura 10. Crecimiento en altura de las plantas en El Carmen, Dgo., Dgo. y Santa Lucía, Pueblo Nuevo, Dgo. a los 6, 12 y 18 meses de plantadas. Letras diferentes para la misma fecha de evaluación indican diferencias significativas (Tukey, $p<0,05$ ). 
mejores crecimientos en el sitio ubicado en Santa Lucía, Pueblo Nuevo, Durango. Un aspecto que influyó positivamente en el crecimiento de las plantas en altura, fue el de las mejores condiciones climáticas de temperatura que prevalecieron con relación al sitio de El Carmen, Durango, Dgo.

En cambio, el crecimiento del diámetro del cuello de las plantas resultó similar estadísticamente $(p>0,05)$ en ambos sitios durante los 18 meses de evaluación (Figura 11). Los mayores incrementos en diámetro del cuello se obtuvieron, en ambos sitios, a los seis meses del plantado con $60,5 \%$ y $67,3 \%$ en El Carmen, Durango, Dgo. y Santa Lucía, Pueblo Nuevo, Dgo., respectivamente; posteriormente, a los 12 meses y 18 meses del plantado, los incrementos en diámetro, con respecto a la evaluación anterior fueron menores al $13,0 \%$.

Escobar et al. (2000), plantaron tres especies en diferentes condiciones de sitio respecto a pendiente y exposición; encontraron que las plantas que en el vivero tenían mayor diámetro, incrementaron más en altura y diámetro en el sitio de plantación. Asimismo, indican que en sitios sin limitantes de humedad el diámetro pierde su capacidad como atributo para predecir la supervivencia.

La producción de fitomasa del sistema radical, de la parte aérea y total resultó estadísticamente igual $(p>0,05)$ en los sitios El Carmen, Durango, Dgo. y Santa Lucía, Pueblo Nuevo, Dgo., por lo que a pesar de las diferencias climáticas entre ambos sitios, éstas no influyeron en la producción de fitomasa en las plantas hasta los 18 meses de plantadas en campo (Figura 12).

La respuesta de las plantas, con excepción de la supervivencia y la altura, debido al efecto del sitio de plantación, resultó similar en ambos sitios, lo cual puede deberse a que el suelo de ambos sitios tiene buena capacidad de reten-

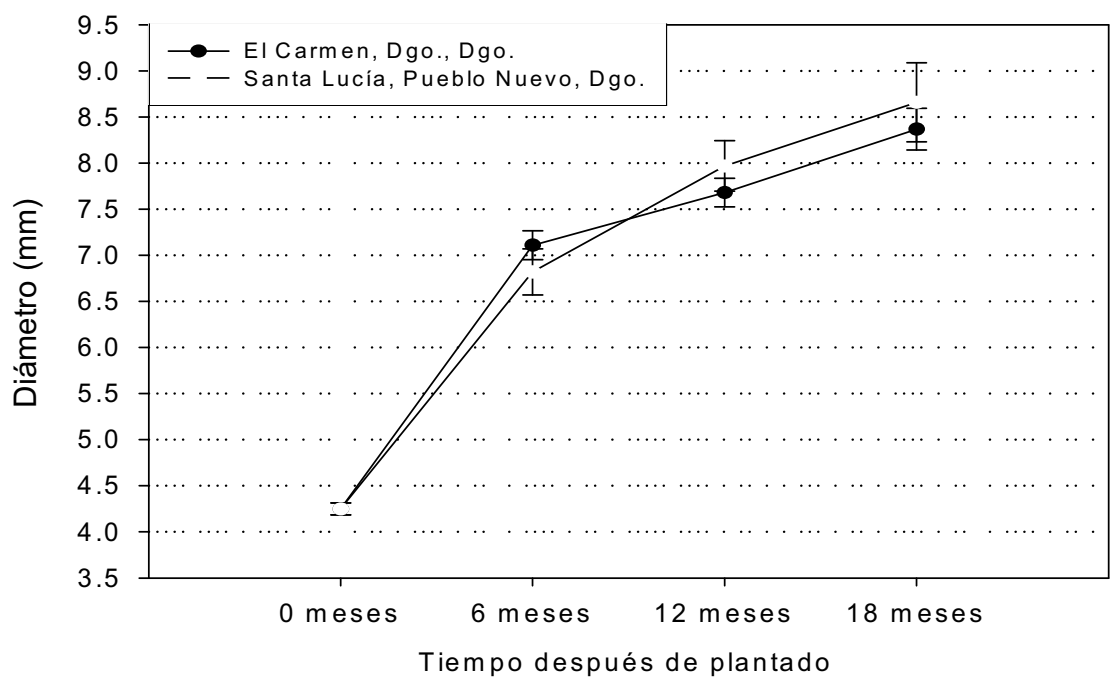

Figura 11. Crecimiento en diámetro del cuello de las plantas en El Carmen, Durango, Dgo. y Santa Lucía, Pueblo Nuevo, Dgo. a los 6, 12 y 18 meses de plantadas. 


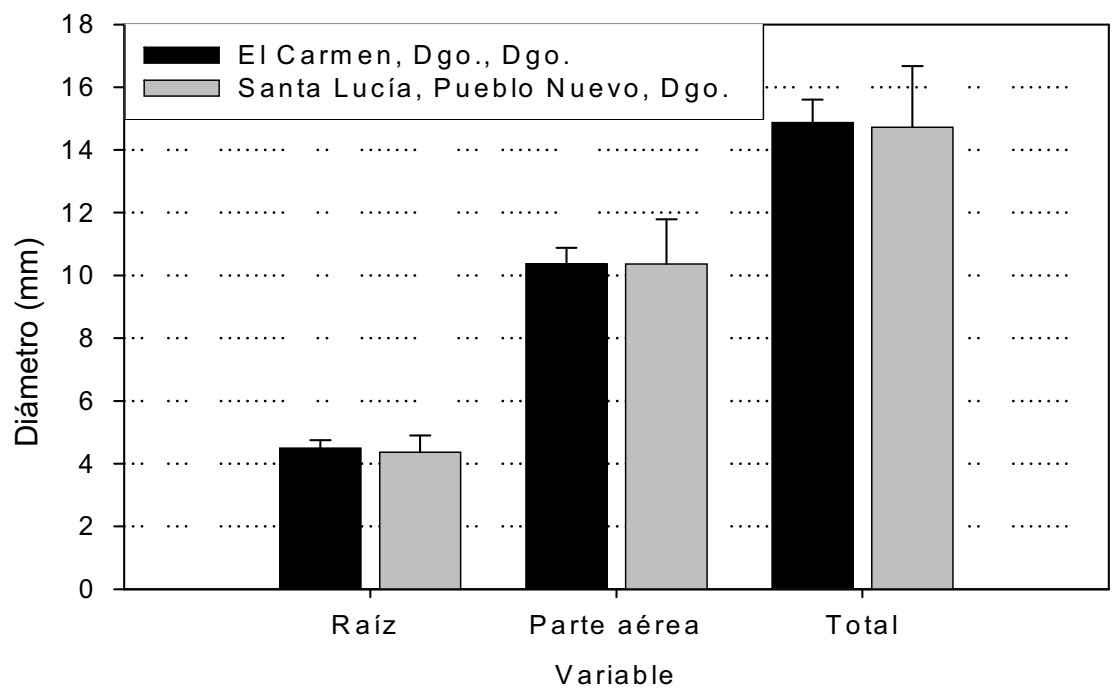

Figura 12. Producción de fitomasa seca de Pinus cooperi Blanco en El Carmen, Durango, Dgo. y Santa Lucía, Pueblo Nuevo, Dgo. a los 18 meses de plantado (febrero de 2004).

ción de humedad y de disponibilidad de nutrimentos, al tener una textura franco arcillosa y franca, poseer un adecuado contenido de materia orgánica, incluso en un nivel alto en el sitio de Santa Lucía, Durango y existir un adecuado nivel de nitrógeno, con una mayor cantidad, en forma de nitrato disponible, en El Carmen, Durango. Con relación al fósforo, éste se considera bajo en el sitio de El Carmen, Durango, mientras que el potasio existe en alta proporción en ambos sitios (Tabla 1). En cambio, como se indicó anteriormente, la humedad disponible y la temperatura que prevalecieron en ambos sitios no fueron factores decisivos para influir y marcar diferencias en los resultados de los sitios evaluados.

\section{DISCUSIÓN GENERAL}

Los resultados obtenidos en los dos sitios plantados, permiten apreciar que la calidad de la planta producida en vivero es importante en su desarrollo posterior en campo; así, en el caso de los tamaños de envase evaluados $\left(80 \mathrm{~cm}^{3}\right.$ y $\left.170 \mathrm{~cm}^{3}\right)$, el envase de $170 \mathrm{~cm}^{3}$ favoreció más la producción de la planta en vivero, efecto que, con excepción de las variables altura y supervivencia, continuó en ambos sitios durante el período de evaluación de las plantas en campo. El tamaño de envase es un elemento trascendental en el proceso de producción de la planta, debido a que define el espacio de crecimiento del sistema radical de las plantas; asimismo, este aspecto adquiere más relevancia cuando se planta en sitios donde existen condiciones adversas de humedad y de calidad del suelo.

Por otro lado, en el factor frecuencia de riegos el tratamiento que más favoreció el crecimiento de las plantas en vivero, riego cada 48 horas, en campo resultó superado, en la mayoría de las variables evaluadas, por el riego cada 96 horas; lo anterior se debe a que la planta 
sin restricción de humedad en la fase de preacondicionamiento de las plantas en vivero resintió las limitantes de humedad existentes en los sitios donde se plantó; en cambio, la planta regada en vivero cada 96 horas, pese a que disminuyó sus tasas de crecimiento al finalizar la etapa de vivero, como respuesta al déficit de humedad, esto le favoreció en campo y se adaptó mejor a la escasez de humedad, lo que le permitió que las plantas tuvieran un diámetro mayor $\mathrm{y}$ produjeran mayor fitomasa.

Finalmente, el factor sitio de plantación influyó en la supervivencia de las plantas y en el crecimiento en altura, con los mejores resultados en supervivencia en el sitio ubicado en El Carmen, Durango. En cambio, el mejor crecimiento en altura se logró en el sitio de Santa Lucía, Pueblo Nuevo, Durango. En el resto de las variables evaluadas los resultados fueron similares. Lo anterior denota que el efecto del sitio de plantación resultó insuficiente para influir en el desarrollo de las plantas, a pesar de que en ambas localidades existieron diferentes condiciones de temperatura y precipitación. Además, las características edáficas de los sitios plantados, en general, presentan apropiadas condiciones para el crecimiento de las plantas y el suelo tiene algunas similitudes en sus características físico químicas.

Para lograr que los programas de reforestación sean exitosos, un aspecto fundamental es la calidad de la planta producida en vivero. Por ello, es necesario que se tengan siempre en cuenta los atributos morfológicos y fisiológicos que deben tener las plantas al salir del vivero, lo cual está definido por varios factores, entre los que destacan los evaluados en este estudio. De esta manera, a futuro puede definirse un sistema de producción que garantice el adecuado establecimiento y crecimiento de las plantas en función de las características de los sitios donde se plante.

\section{CONCLUSIONES}

La planta producida en el envase de $170 \mathrm{~cm}^{3}$ tuvo mejor calidad en la fase de campo durante los 18 meses de evaluación. Sobresalieron las variables diámetro y producción de fitomasa, parámetros importantes en el crecimiento futuro de las plantas debido a que son indicadores definitorios de la calidad de la planta producida en vivero.

Aunque el preacondicionamiento de las plantas en vivero, mediante la restricción de humedad, no mostró resultados contundentes en la fase de campo, existió una tendencia favorable para la frecuencia de riego cada 96 horas. Ahí se encontraron las mejores respuestas en el crecimiento de las plantas en altura y producción de fitomasa de la raíz, de la parte aérea y total, a los 18 meses de plantado.

Las condiciones climáticas y edáficas de los sitios evaluados sólo influyeron en la supervivencia y en el crecimiento en altura de las plantas, durante los 18 meses de evaluación. La planta establecida en el sitio El Carmen, Durango, Dgo., tuvo un $19,6 \%$ más de supervivencia con relación al otro sitio de plantación. En cambio, en el sitio de Santa Lucía, Pueblo Nuevo, Dgo., sobresalió el crecimiento en altura con una diferencia cercana a los $4 \mathrm{~cm}$.

\section{RECONOCIMIENTOS}

Al INIFAP y al CONACyT, por la beca otorgada al primer autor para realizar estudios de Doctorado. A la Fundación Produce Durango, A.C., por el financiamiento brindado a través del Proyecto 
Red de Bosques Modelo Durango. A los técnicos del INIFAP: C. Francisco J. Falcón F. y Francisco J. Chávez O., por su valioso apoyo en la toma de datos. Al Ejido Pueblo Nuevo y a los propietarios del Predio Particular El Carmen, quienes a través de su personal técnico apoyaron en el trabajo de campo.

\section{REFERENCIAS}

Aldana B., R. y M. Aguilera R. 2002. Procedimientos y cálculos básicos útiles en la operación de viveros que producen en contenedor. PRONARE. CONAFOR. Documento técnico. Guadalajara, Jal. 44 p.

Bainbridge, D. A. 1994. Container optimization-field data support container innovation. In: Landis, T.D., and R.K. Dumroese (tech. coords.). Gen. Tech. Rep. RM-GTR-257. Fort Collins, Co. USDA. Forest Service. pp: 99-104

Barnett, J. P. y M. J. McGilvray. 2000. Growing longleaf pine seedlings in containers. Native Plants Journal 1(1): 54-58.

Barnett, J. P. 2002. Longleaf: Why plant it? Why use containers? In: Barnett, J.P.; Dumroese, R. K., and D. J. Moorhead (eds.). Proceedings of workshops on growing longleaf pine in containers 1999 and 2001. Gen. Tech. Rep. SRS-56. Asheville, N.C. USDA. Forest Service, Southern Research Station. 63 p.

Beyeler, J. 1996. Effect of planting methods on field performance of black spruce five years after planting. Forest Research Report 62. Cooperation Agreement for Forestry Development. Nova Scotia. Department of Natural Resources. Nova Scotia, Canada.
Birchler, T., R. W. Rose, A. Royo y M. Pardos. 1998. La planta ideal: Revisión del concepto, parámetros definitorios e implementación práctica. Invest. Agr. Sist. Recur. For. 7(1 y 2): 110-121.

Boivin, J. R., B. D. Miller y V. R. Timmer. 2002. Late-season fertilization of Picea mariana seedlings under greenhouse culture: biomass and nutrients dynamics. Ann. For. Sci. 59: 255-264.

Cañellas, I., L. Finat, A. Bachiller y G. Montero. 1999. Comportamiento de planta de Pinus pinea en vivero y campo: Ensayos de técnicas de cultivo de planta, fertilización y aplicación de herbicidas. Invest. Agr.: Sist. Recur. For. 8(2): 335-359.

Carlson, W. C. y D. E. Miller. 1990. Target seedling root system size, hydraulic conductivity and water use during seedling stablishment. In: Rose, R., S.J. Campbell, and T.D. Landis (eds.). Target Seedling Symposium: Proceedings, Combined Meeting of the Western Forest Nursery Associations. August 13-17, 1990. Gen. Tech. Rep. RM-200. Fort Collins, Co. USDA. Forest Service. pp: 53-66.

Cetina A., V. M., V. A. González H., M. L. Ortega D., J. Vargas H. y A. Villegas M. 2002. Supervivencia y crecimiento en campo de Pinus greggii Engelm., previamente sometido a podas o sequía en vivero. Agrociencia 36: 233-241.

CONAFOR. 2004a. Programa Nacional para el Manejo de los Recursos Genéticos Forestales (PNMRGF). SEMARNAT-CONAFOR. Zapopan, Jalisco. $35 \mathrm{p}$.

CONAFOR. 2004b. Evaluaciones PRONARE 2000, 2001. [En línea 
http://www.conafor.gob.mx/program as_nacionales_forestales/evaluaciones/index.html. Julio 2004].

Cornejo O., E. y W. Emmingham. 2003. Effects of water stress on seedling growth, water potential and stomatal conductance of four Pinus species. Crop. Res. 25(1): 159-190.

Domínguez L., S., I. Carrasco M., N. Herrero S., L. Ocaña B., J. L. Nicolás P., y J. L. Peñuelas R. 2000. Las características de los contenedores influyen en la supervivencia y crecimiento de plantas de Pinus pinea en campo. Actas del Primer simposio sobre el pino piñonero. Valladolid, España. pp: 203-209.

Escobar R., R., C. Bassaber E., M. Sánchez O. y A. Escobar S. 2000. Efectos de la exposición, ubicación de la pendiente y calidad de plantas en tres especies de interés forestal en Chile. Primer Congreso Nacional de Reforestación. CONAFOR. Colegio de Posgraduados. Montecillos, México. $10 \mathrm{p}$.

Landis, T. D. 2002. The target seedling concept. A tool for better comunication between nurseries and their customeers. In: Riley, L.E., R.K. Dumroese, and T.D. Landis (tech. coords.) National Proceedings: Forest and Conservation Nursery Associations. 2002. Proceedings. RMRS-P-28. Odgen, UT. USDA. Forest Service, Rocky Mountain Research Station. pp: 31-36.

Martínez T., T., J. J. Vargas H., A. Muñoz O. y J. López U. 2002. Respuesta al déficit hídrico de Pinus leiophylla: Consumo de agua y crecimiento en plántulas de diferentes poblaciones. Agrociencia 36: 365-376.
Mexal, J. G. y T. D. Landis. 1990. Target seedlings concepts: Height and diameter. In: Rose, R., S.J. Campbell, and T.D. Landis (eds.). Target Seedling Symposium: Proceedings, Combined Meeting of the Western Forest Nursery Associations. August 13-17, 1990. Gen. Tech. Rep. RM200. Fort Collins, Co. USDA. Forest Service. pp: 17-36.

Moreno T., R.E. 2000. Observaciones de un viverista y productor de planta forestal en la región del Pacífico Noroeste de Estados Unidos. Primer Congreso Nacional de Reforestación. Dirección General del Programa Nacional de Reforestación. SEMARNAP. Colegio de Posgraduados. Montecillo, México. 6 p.

Mullan, G. D. y P. J. White. 2002. Seedling quality: Making informed choices. Bushcare and the Department of Conservation and Land Management W.A. Wheatbelt Region.

Prieto R., J.A., E.H. Cornejo O., P.A. Domínguez C., J.J. Návar $\mathrm{CH}$., J.G. Marmolejo M. y J. Jiménez P. 2004. Estrés hídrico en Pinus engelmanii Carr., producido en vivero. Invest. Agr.: Sist. y Rec. For. 13(3): 443-451.

Scagel, R., M. Madill, C. Kooistra y R. Bowden. 1998. Provincial seedling stock type selection and ordering guidelines. Province of British Columbia. Ministry of Forests. Canada. $71 \mathrm{p}$.

South, B. D., W. S. Harris, P. J. Barnett, J. M. Hainds y H. D. Gjerstad. 2005. Effect of container type and seedling size on survival and early height growth of Pinus palustris seedlings in Alabama, U.S.A. Forest Ecology Management 204(2-3): 385-398. 
Universidad Autónoma de Tlaxcala. 2002. Programa Nacional de Reforestación. Ejercicio 2002. Durango. CONAFOR. Coordinación General de Conservación y Restauración. Coordinación General de Planeación e Información. PRONARE. Zapopan, Jalisco. $92 \mathrm{p}$.

Villar S., P., L. Ocaña B., J.L. Peñuelas R., I. Carrasco M. y S. Domínguez L. 1997. Efecto de diferentes niveles de endurecimiento por estrés hídrico en el contenido de nutrientes y la resistencia a la desecación de plántulas de Pinus halepensis L. Actas del II Congreso Forestal Español. pp: 673-678.
Villar S., P., S. Garrachón, S. Domínguez L, J.L. Peñuelas R., R. Serrada y L. Ocaña. 2001. Desarrollo en campo, arquitectura radical y estado hídrico seis años después de la plantación de brinzales de Pinus pinea cultivados en diferentes tipos de contenedores. Actas del III Congreso Forestal Español. Granada, España. pp: 797-803.

Manuscrito recibido el 31 de mayo de 2005

Aceptado el 28 de agosto de 2006.

Este documento debe citarse como: Prieto Ruiz, J. A., P. A. Domínguez Calleros, E. H. Cornejo Oviedo y J. J. Návar Cháidez. 2007. Efecto del envase y del riego en vivero en el establecimiento de Pinus cooperi Blanco en dos condiciones de sitio. Madera y Bosques 13(1):79-97. 
